Chapter 8

\title{
Demodulation of FM Data in \\ Free-Space Optical Communication Systems \\ Using Discrete Wavelet Transformation
}

\author{
Nader Namazi, Ray Burris, G. Charmaine Gilbreath, \\ Michele Suite and Kenneth Grant
}

Additional information is available at the end of the chapter

http://dx.doi.org/10.5772/52433

\section{Introduction}

Free-space optical (FSO) communications links have the potential to deliver very high bandwidth due to the high carrier frequency as compared with RF links. They have the advantages of being rapidly deployable and less expensive to install than optical fiber systems. The low divergence of laser beams means that FSO systems are intrinsically low in probability of intercept in comparison to RF, and being 'line-of-sight' avoids wasteful use of both the frequency domain (bandwidth allocation) and the spatial domain. Another advantage of FSO communication links over RF communications is the large unregulated bandwidth as compared with the heavy traffic and expensive bandwidth allocations for RF links.

However, one of the main factors reducing SNR in FSO communications is scintillation noise due to turbulence. Atmospheric turbulence produces temporary pockets of air with slightly different temperatures and pressures, and therefore with slightly different indices of refraction. These turbulence cells act as small, weak lenses that refract the light slightly and cause distortions in the wave front as a laser beam propagates through the atmosphere. The resulting variation in the arrival time of various components of the beam produces constructive and destructive interference at the receiver, causing fluctuations in laser beam intensity. These rapid fluctuations are known as scintillation and occur on a time scale comparable to the time it takes these cells to move across the beam path due to the wind (typically on the order of a millisecond). These intensity fluctuations become amplitude fluctuations in the case of analog modulation of the laser beam. In addition, atmospheric turbulence can cause beam break-up and beam wander which can cause very large swings in the average received power, on the 
order of tens of $\mathrm{dB}$, on frequency scales from dc up to several kilohertz. This causes the average received AC signal to not be clamped at zero due to inadequate AC coupling.

There are many applications in which data is collected from an analog sensor or system and transmitted long distances to the end user. Typically the data would subsequently be digitized and transmitted over an RF or fiber optic communication link. Problems occur if the platform containing the sensor system is size, weight, or power (SWaP) constrained, since high speed digitizers can greatly add to the SWaP burden. Also, if the data or required communication is of a sensitive nature and secure communication links are not available, the user runs the risk of having the communication detected and/or intercepted. In these cases, it would be of great benefit to have the capability of transmitting unprocessed analog sensor data over a secure channel. Free-space lasercomm using analog or RF modulation of the transmitted laser beam can provide a method for transmitting un-digitized data over a high speed communication link that has a very low probability of detection and intercept, as well as being highly resistant to jamming efforts due to the relatively narrow field-of-view of the receivers. However, atmospheric turbulence as discussed above makes this process problematic.

Methods to correct the aberrations caused by atmospheric turbulence and to thus enable transmission of analog data over a FSO link are currently being explored. This work deals with scenarios in which a frequency-modulated waveform is transmitted through an FSO channel. Several applications of the DWT are employed in the receiver end to demodulate the transmitted data.

The chapter is organized as follows. Section 2 reviews recent advances in using analog FM to transmit data over the free space channel. Section 3 describes the mathematical modeling of the received FSO signal. Section 4 is dedicated to de-noising of the FSO signal using the DWT and Section 5 is devoted to the simulation experiments. Finally, we present the summary and conclusions in Section 6.

\section{Applications}

The transmission of RF modulated laser beams through optical fibers and the characterization of the information transmitted have been the subject of research for many years [1-3]. More recently, however, the potential advantages of the free space channel have led to research into its use as a medium for transmission of RF analog data. Refai et al. [4] undertook a comparative study of fiber optic links and FSO links. They concluded that FSO is suitable for RF transmissions; that it can perform comparably with fiber-based links; and that FSO can be an attractive substitute for fiber optic links when a clear line-of-sight is available. Bucholtz et al. [5] performed a statistical study of RF analog FSO links. In fiber-based systems, most of the significant parameters such as RF gain, noise figures, and linearity can, in the absence of component degradation or change, be treated as constants. In FSO systems, on the other hand, these parameters are not constant. In particular, the received power can vary by tens of decibels due to atmospheric turbulence. They reported that the link parameters of gain, noise factor and third-order spurious free dynamic range depend entirely on the statistics of the received optical power. 
Since 2005, there have been several reports in the literature on demonstrations of FSO analog links, with increasing range and performance. In a bench top demonstration, Refai et al. [6] transmitted cable TV signals using wavelength division multiplexing. This was done with a view to eventual deployment in "last mile" situations. Murphy et al. [7] described an optical link using a modulating retro-reflector (MRR) [8]. The laser beam was encoded with a FM signal of carrier frequency $\sim 750 \mathrm{kHz}$, and successfully transmitted an audio signal over bench top distances.

Analog modulation has been successfully applied to FSO transmission of video signals. Baseband AM provides optimum use of bandwidth, and transmission of composite video has been demonstrated using amplitude modulation [9], although this suffers from signal degradation due to atmospheric scintillation. A technique employing dual wavelengths has been demonstrated to be effective in mitigating scintillation noise by using common mode rejection to remove co-channel noise $[10,11]$, but the utility of this is limited by the complexity of the system and linearity constraints in the amplitude domain. This constraint was removed by using frequency modulation of a sub-carrier to transmit audio/video signals over a $1.5 \mathrm{~km}$ terrestrial path $[12,13]$. This work has now been extended to include bidirectional audio transmission, and has been demonstrated at ranges up to $3 \mathrm{~km}$ in the maritime environment using a modulating retro-reflector [14]. Burris et al. showed analog FM to be effective in long range links, by transmitting audio/video signals over a folded $32 \mathrm{~km}$ maritime path [15].

\section{Mathematical modeling of received FSO signal}

The received FSO signal can be described as

$$
r(t)=x_{F M}(t) \sigma(t)+m(t)+w(t)
$$

in which $x_{F M}(t)$ characterizes the frequency-modulated signal and $\sigma(t)$ signifies the atmospheric scintillation noise. In addition, the model (1) assumes two types of additive noise. The first noise component, $m(t)$, is the relatively low-frequency fluctuations of the signal mean value caused by insufficient AC-coupling. The second additive term $w(t)$, portrays the additive white Gaussian noise (AWGN) with zero-mean. Furthermore the frequency-modulated waveform is formed as,

$$
x_{F M}(t)=A \cos \left[\omega_{c} t+k_{f} \int_{-\infty}^{t} \mathrm{~d}(\alpha) d \alpha\right]
$$

in which $d(t)$ represents the information (analogue) data, $A$ is a gain, $k_{f}$ is the modulation index and $\omega_{c}$ is the carrier frequency [16]. 
In the following section we use the Discrete Wavelet Transformation (DWT) to process $r(t)$ for noise reduction.

\section{De-noising of FSO signal using discrete wavelet transform}

This section deals with the application of the Discrete Wavelet Transformation (DWT) to the de-noising of the received FSO waveform $r(t)$ expressed in (1).

The DWT is a powerful iterative technique for decomposition of a signal into approximation (low frequency) and detail (high frequency) waveforms [17]. The process begins by decomposing the coefficients of the first level of decomposition of the signal into coefficients of approximation, $c A_{1}$, and coefficients of detail, $c D_{1}$. Accordingly, the coefficients $c A_{1}$ are further decomposed into $c A_{2}$ and $c D_{2}$ to generate the second level of decomposition. The process can continue for the $i^{\text {th }}$ level of decomposition for which $c A_{i}$ and $c D_{i}$ are evaluated from $c A_{i-1}$. At each level, the DWT coefficients can be used to reconstruct the approximation and the detail of the original signal. Figure 1 illustrates three levels of decomposition of the DWT coefficients.

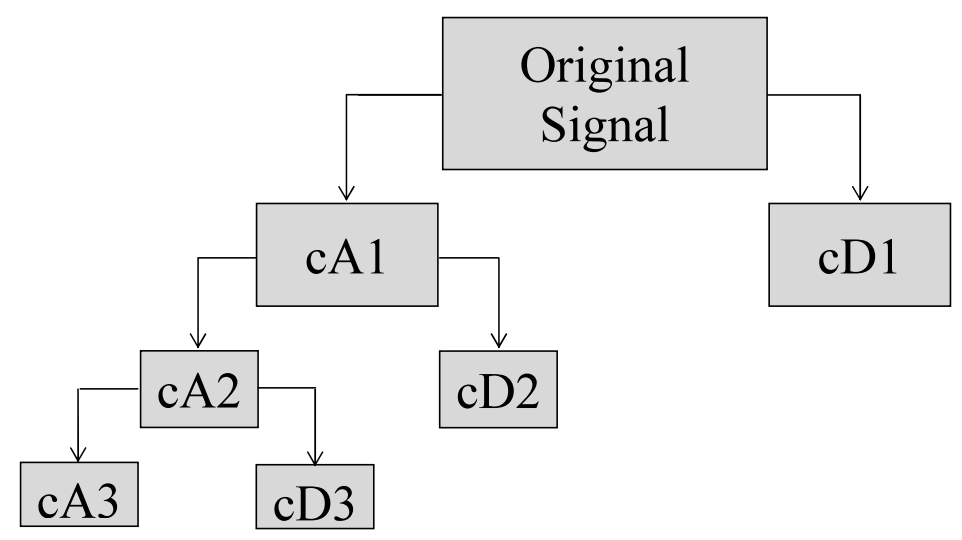

Figure 1. A Three Level Decomposition of the DWT Coefficients

A specific strength of the DWT is its ability to decompose a signal into low-frequency and highfrequency waveforms at any desired level. This property can be directly applied into the received FSO waveform of (1) in order to identify and remove the unwanted low-frequency signal $m(t)$ and the undesirable low-frequency scintillation waveform $\sigma(t)$. Moreover, the energy of the high-frequency component of the white noise $w(t)$ can be considerably reduced using the decomposition property of the DWT.

The process of removing the low-frequency noise $m(t)$ is performed in two consecutive steps. We first find the approximation of $r(t)$ in an appropriate level to obtain $\hat{m}(t)$. We consequently form a subtraction process as follows: 


$$
r_{1}(t)=r(t)-\hat{m}(t)
$$

Hence, the received FSO signal (1) after cancellation of $m(t)$ becomes

$$
r_{1}(t)=x_{F M}(t) \sigma(t)+w_{1}(t)
$$

where $w_{1}(t)$ is the noise term that includes $w(t)$ as well as the error caused due to the determination of $m(t)$. The next step deals with the cancellation of the low frequency scintillation noise $\sigma(t)$. As an intermediate step, it is conceivable to form the square of the new signal shown in (4); that is,

$$
r_{2}(t)=r_{1}^{2}(t)=x_{F M}^{2}(t) \sigma^{2}(t)+w_{1}^{2}(t)+2 w_{1}(t) x_{F M}(t) \sigma(t)
$$

Application of (2) in (5) results in a low-frequency signal $\frac{A^{2} \sigma^{2}(t)}{2}$ plus a collection of highfrequency signals shown by HF:

$$
r_{2}(t)=\frac{A^{2} \sigma^{2}(t)}{2}+H F
$$

It is observed from (6) that the square process has enhanced the difference between the low and high frequency components of the received signal; hence, it is more effective to use DWT for signal separation. Subsequently, by finding the DWT approximations of $r_{2}(t)$ in an appropriate level, $\hat{\sigma}(t)$ after a square root device, can be determined. To continue, multiply $r_{1}(t)$ in (4) by the inverse of $\hat{\sigma}(t)$; hence,

$$
r_{3}(t)=\frac{r_{1}(t)}{\hat{\sigma}(t)}=x_{F M}(t)+w_{3}(t)
$$

where in $(7)$ it is assumed that $w_{3}(t) \triangleq w_{1}(t) / \hat{\sigma}(t)+\varepsilon, \varepsilon$ is an error due to the approximation of $\sigma(t)$ and $\hat{\sigma}(t) \neq 0$. The FM signal $\hat{x}_{F M}(t)$ can be finally demodulated using any conventional FM demodulator to provide the analog data $\hat{d}(t)$. The noisy waveform $\hat{d}(t)$ c्रan be further de-noised using an additional application of the DWT. This signal is denoted as $\hat{d}_{D N}(t)$. It is noticed that we have de-noised $\hat{d}(t)$ not $r_{3}(t)$. This is due to the fact that the demodulated message $\hat{d}(t)$ is characteristically a baseband waveform which can be de-noised more effectively than the relatively high-frequency waveform $r_{3}(t)$. Figure 2 illustrates the entire process. 


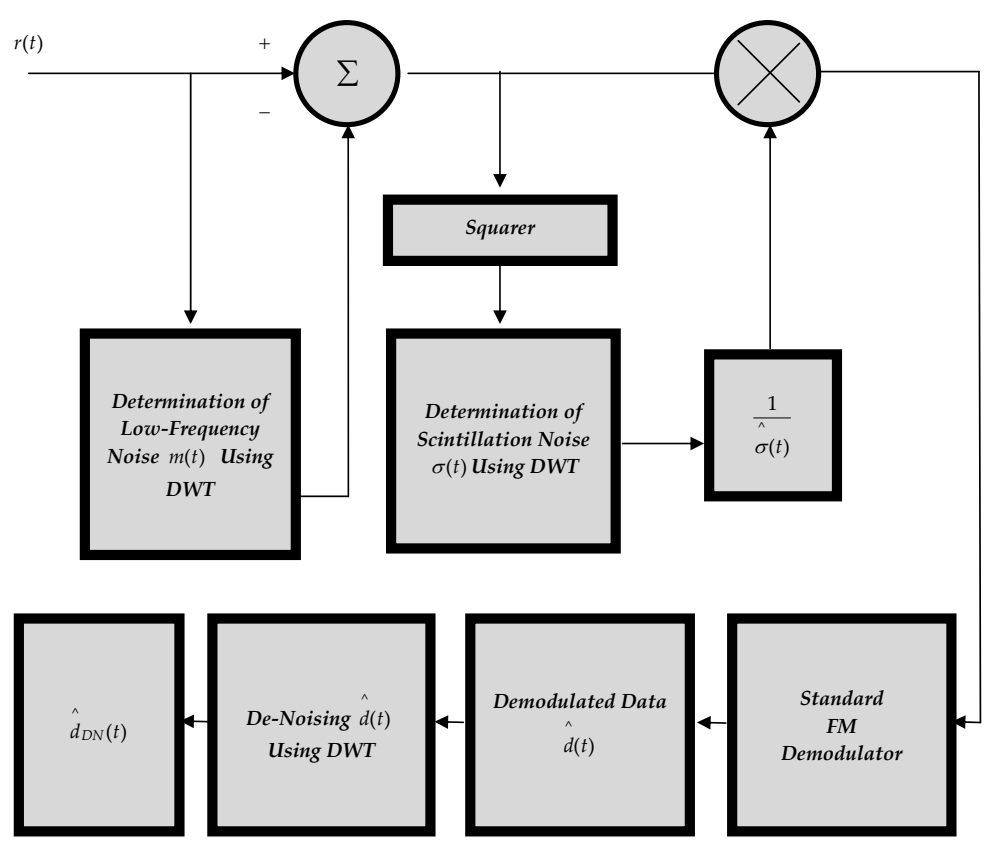

Figure 2. Structure of the FM/FSO Receiver

\section{Simulation experiments}

This section presents the results of simulation experiments. We present the results in two sets of experiments. Experiment I uses a 1-D time signal and deals with the sensitivity of the algorithm to the variations of $S N R$ and $S V$ (defined below). This experiment is primarily presented for quantitative evaluations of the method. Experiment II employs a 2-D single image as the original data and is mainly focused on the qualitative assessment of the algorithm.

\section{Experiment I}

In this experiment the received waveform (1) was synthesized by generating an FM signal with the carrier frequency $\omega_{c}=2 \pi \times(1.36 \mathrm{MHz})$. The assumed data $d(t)$ was used as follows:

$$
d(t)=-0.2 \cos \left(\omega_{d 1} t+\pi / 16\right)+0.3 \sin \left(\omega_{d 2} t-\pi / 8\right)-0.1 \sin \left(\omega_{d 3} t\right)
$$

with $\omega_{d 1}=2 \pi \times 60000 ; \omega_{d 2}=2 \pi \times 30000 ; \omega_{d 3}=2 \pi \times 10000$. The sampling radian frequency was assumed to be $5 \times \omega_{c}$. In addition, the noise signals $\sigma(t)$ and $m(t)$ were duplicated from a real FSO channel and the AWGN noise $w(t)$ was synthetically generated in MATLAB. The data 
was processed in one frame of 990,000 sample points. The low frequency noise $m(t)$ was extracted as shown in (3) using the DWT with the Daubechies 20 (db20) mother wavelet and 6 decomposition levels. Also the desired signal in (6) was separated using db20 mother wavelet with 10 decomposition levels. In addition, the demodulated message $d(t)$ was de-noised using DWT with db20 mother wavelet and 3 decomposition levels. The Signal-to-Noise Ratio (SNR) was defined as

$$
S N R=10 \log _{10}\left(\frac{\sigma_{d}^{2}}{\sigma_{w}^{2}}\right)
$$

where $\sigma_{d}^{2}$ is the variance of the data $d(t)$ and $\sigma_{w}^{2}$ is the variance of $w(t)$ in (1). To study the sensitivity of the algorithm to the scintillation changes we define the Scintillation Variation (SV) parameter as

$$
\mathrm{SV}=20 \log _{10}\left[\frac{\sigma_{\text {max }}(\mathrm{t})}{\sigma_{\text {min }}(\mathrm{t})}\right]
$$

This quantity is a measure of the abrupt variation of the scintillation noise $\sigma(t)$.

Figures 3 through 9 represent the results of this experiment. Figure 3 illustrates the FM/FSO signal $r(t)$ represented by Equations (1) and (2). Figure 4 highlights, for SNR = $0 \mathrm{~dB}$, the frequency descriptions of the transmitted FM signal, the received FSO/FM waveform, and the processed FM signal after removal of $m(t)$ and $\sigma(t)$. The middle figure in this set indicates that the spectrum of the FSO/FM waveform carries a relatively large amount of low-frequency components. This is primarily due to the presence of the slowly-varying terms $m(t)$ and $\sigma(t)$. It is shown in Figure 4 that the DWT is quite successful in reshaping the spectrum of the FSO/FM signal from the middle figure to the one shown at the bottom figure. Figure 5 highlights, for $S N R=0 \mathrm{~dB}$, the time history of the transmitted FM signal and the processed FM signal after extracting $m(t)$ and $\sigma(t)$. Figure 6 displays a close-up views of the transmitted message $d(t)$, demodulated message $d(t)$, and de-noised demodulated message $d_{D N}(t)$ for SNR $=0 \mathrm{~dB}$. This figure indicates that the original data $d(t)$ is closely extracted from the FSO/FM signal. Further, the de-noising of the demodulated data appears to be quite effective. Figure 7 displays, for $\mathrm{SNR}=20 \mathrm{~dB}$, the transmitted FM signal and the processed FM signal after removing $m(t)$ and $\sigma(t)$. Figure 8 displays a close-up views of the transmitted message $d(t)$, demodulated message $d(t)$, and de-noised demodulated message $d_{D N}(t)$ for SNR $=20 \mathrm{~dB}$. Figure 9 shows Mean-Square Error for the message $d(t)$ versus SV for various levels of SNR. This figure highlights the important result that the performance of the algorithm is nearly identical under various SV values and for fixed SNR. In other words, the method tends to be insensitive to the variations of the scintillation noise, especially for large levels of SV. 


\section{Experiment II}

As a demonstration of the efficiency of this algorithm, we consider the situation in which the transmitted message $d(t)$ is the row-ordered vector of the still image shown in Figure 10. The scintillation variation, $\mathrm{SV}$, is fixed to $20 \mathrm{~dB}$ in this experiment. Similar to Experiment $\mathrm{I}$, the data is frequency modulated using $\omega_{c}=2 \pi \times(1.36 \mathrm{MHz})$. Figures 11, 12 and 13, respectively, highlight the demodulated FM/FSO signal for $\mathrm{SNR}=10 \mathrm{~dB}$, and $\mathrm{SNR}=20 \mathrm{~dB}$ and $\mathrm{SNR}=50 \mathrm{~dB}$. It is seen from these figures that as the SNR improves, the performance consistently improve.

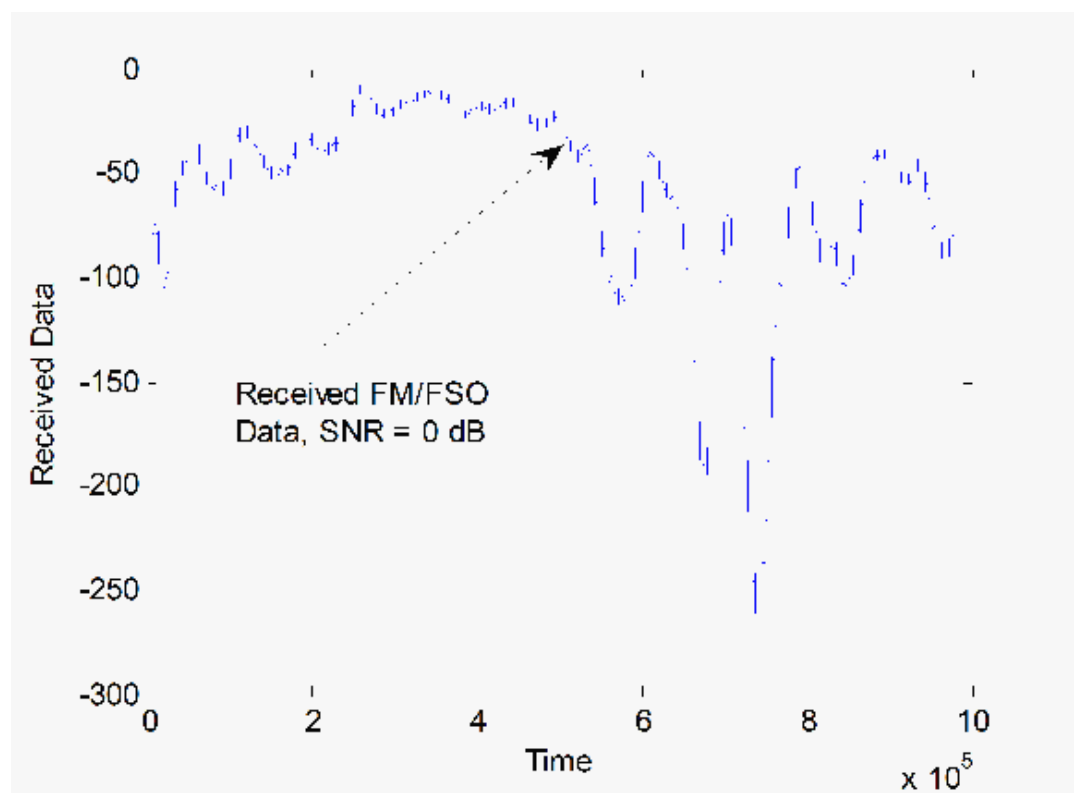

Figure 3. A Typical FM/FSO Signal. 

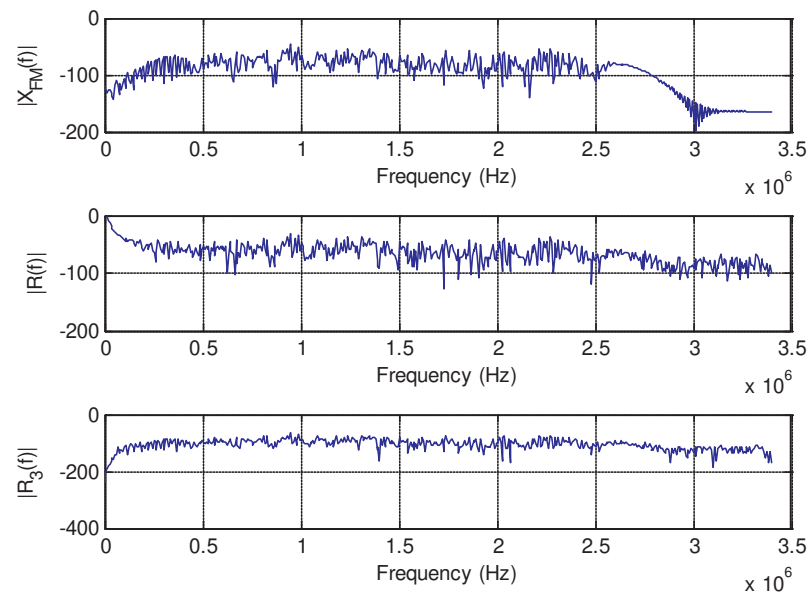

Figure 4. From Top to Bottom and for $S N R=0 \mathrm{~dB}$ : The Absolute Amplitude Spectrum of Transmitted FM signal, $X_{F M}(f)=\mathfrak{J}\left\{X_{F M}(t)\right\}$, The Absolute Amplitude Spectrum of Received Noisy FSO/FM Signal, $R(f)=\mathfrak{J}\{r(t)\}$ shown in Figure 3 , The Absolute Amplitude Spectrum of Processed FM Signal after removal of $m(t)$ and $\sigma(t), R_{3}(f)=\mathfrak{T}\left\{r_{3}(t)\right\}$, where $r_{3}(t)$ is defined in (7).
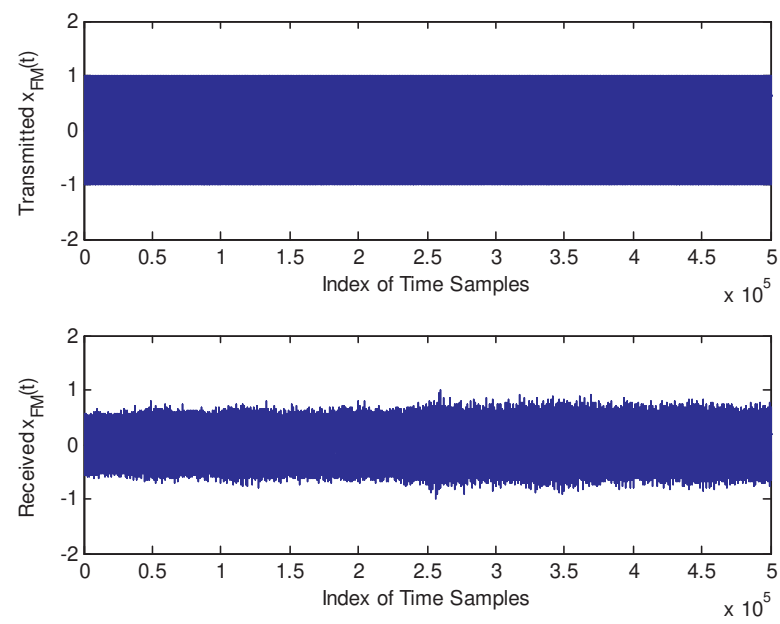

Figure 5. Top: The Transmitted FM Signal, $x_{F M}(t)$, Bottom: The Processed FM Signal after Extracting $m(t)$ and $\sigma(t)$ for $\mathrm{SNR}=0 \mathrm{~dB}$. 


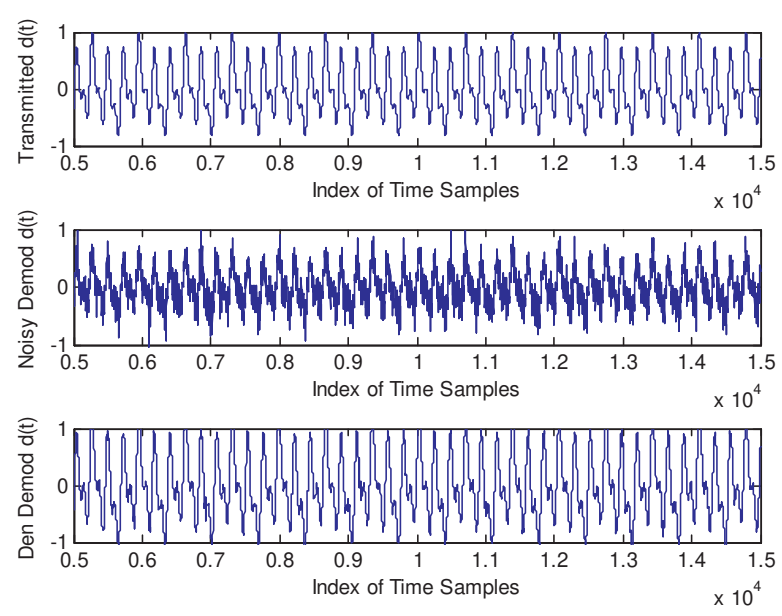

Figure 6. From Top to Bottoxm, SNR =0, Close-Ups of: Transmitted Message $d(t)$, Demodulated Message $\hat{d}(t)$, De-noised Demodulated Message $d_{D N}(t)$.
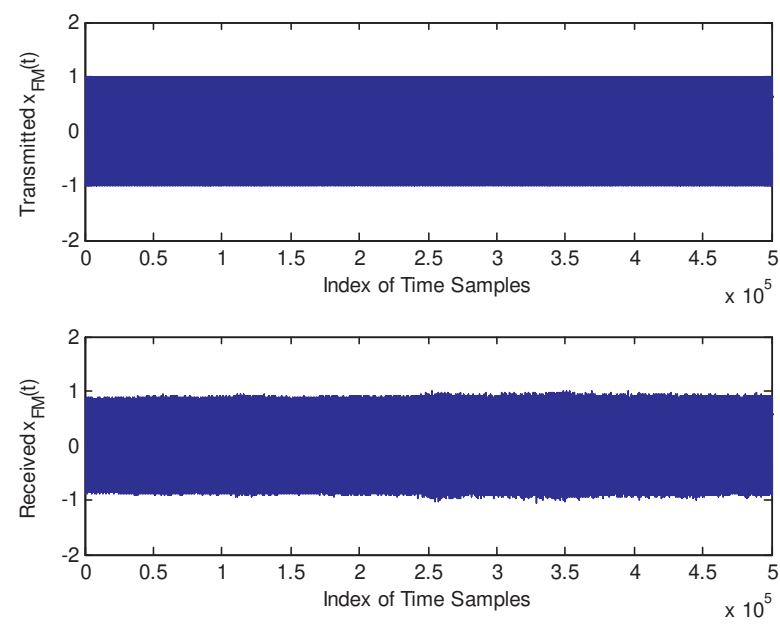

Figure 7. Top: The Transmitted FM Signal, $x_{F M}(t)$, Bottom: The Processed FM Signal after Extracting $m(t)$ and $\sigma(t)$ for $S N R=20 \mathrm{~dB}$. 


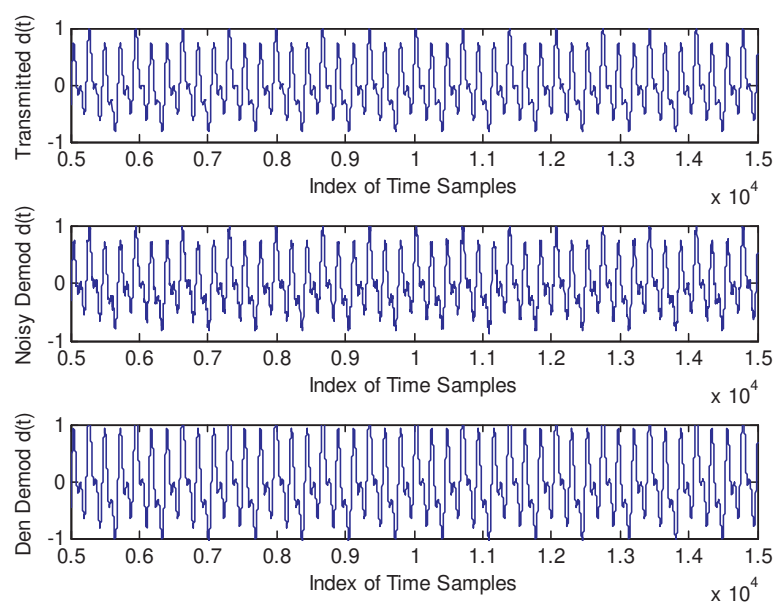

Figure 8. From Top to Bottom $\operatorname{SNR}=20$, Close-Ups of: Transmitted Message $d(t)$, Demodulated Message $\hat{d}(t)$, Denoised Demodulated Message $d_{D N}(t)$.

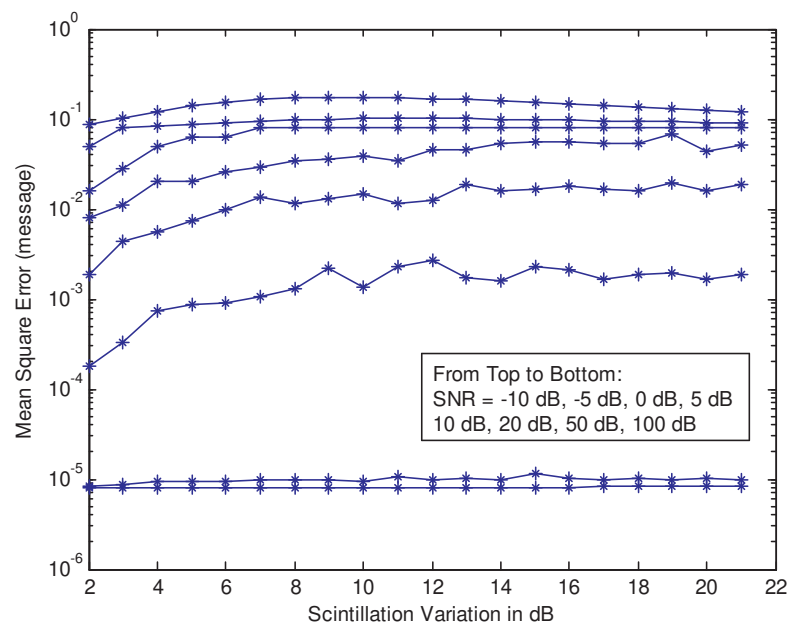

Figure 9. Mean-Square Error versus SV for various levels of SNR 


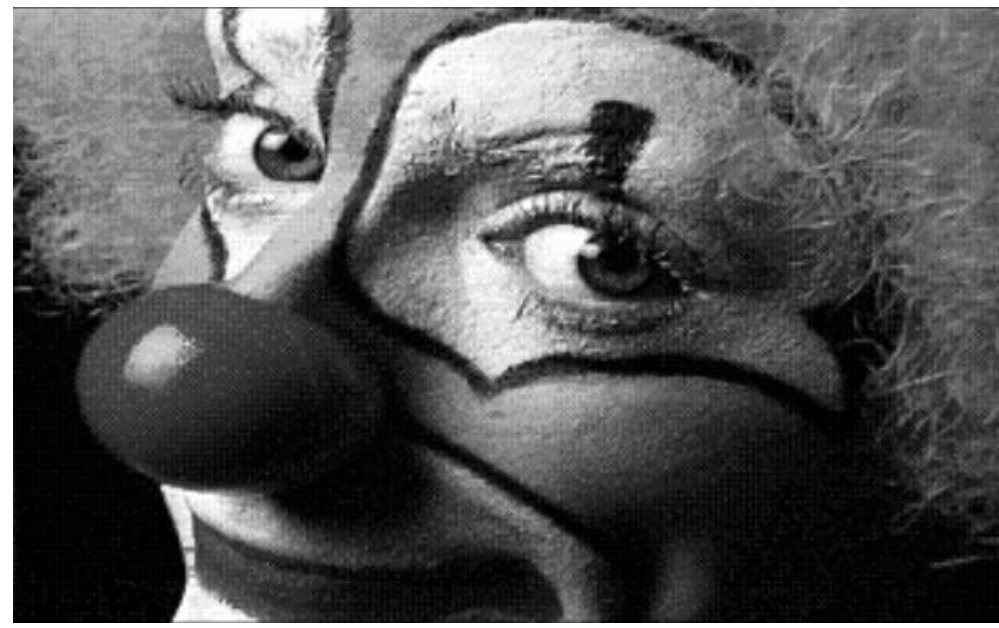

Figure 10. Transmitted Image

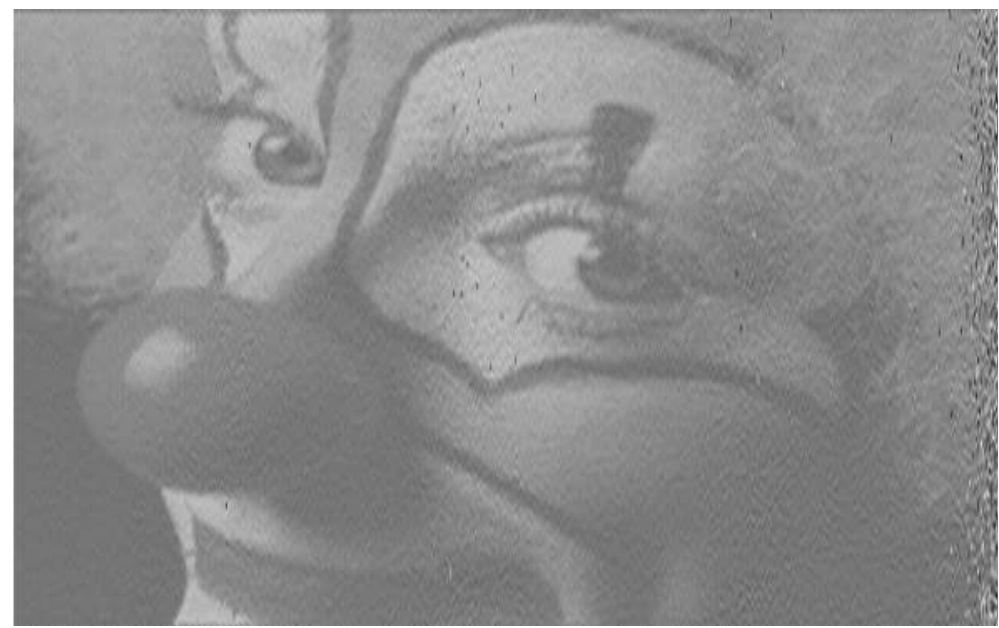

Figure 11. De-noised demodulated Image, $S N R=10 \mathrm{~dB}, \mathrm{SV}=20 \mathrm{~dB}$ 


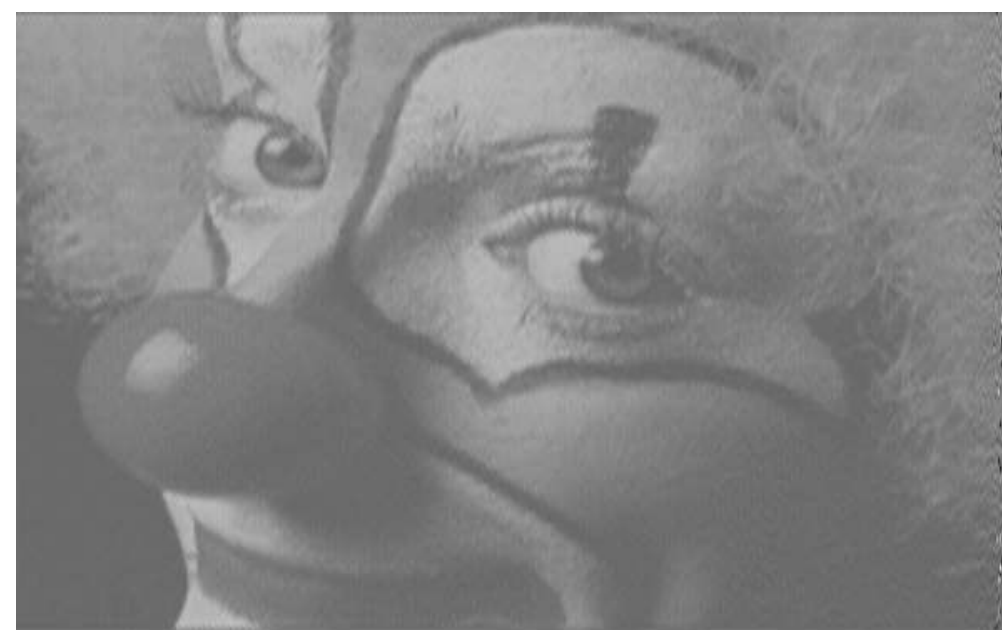

Figure 12. De-noised demodulated Image, $S N R=20 \mathrm{~dB}, \mathrm{SV}=20 \mathrm{~dB}$

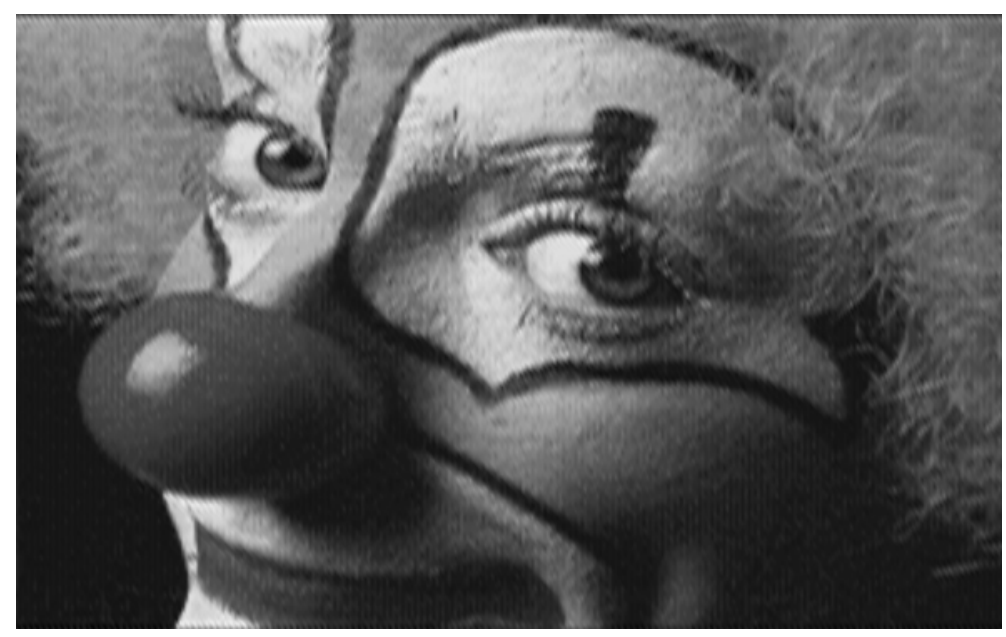

Figure 13. De-noised demodulated Image, $S N R=50 \mathrm{~dB}, \mathrm{SV}=20 \mathrm{~dB}$ 


\section{Summary and conclusions}

Atmospheric noise signals are a fundamental limitation of free-space optical communications. In this work we presented the limitations that this imposes, and investigated the use of the discrete wavelet transformation (DWT) to overcome them. Simulation experiments were performed to validate the use of the DWT in the demodulation of the FM data in the presence of scintillation noise, noise due to insufficient AC-coupling, and AWGN. It was demonstrated that the use of the DWT, as explained in the paper, is quite effective in reducing the joint effects of the atmospheric as well as the additive white Gaussian noises.

Several concluding remarks are in order. It is noted that despite the fact that FM was the modulation type presented in this paper, our algorithm can be extended to other constantenvelope (digital or analog) modulation scheme. This stems from the fact that in constantenvelope modulations, the message is solely modulating the phase of the carrier. Consequently, any changes in the magnitude of the received FSO signal are exclusively due to the noise terms, $m(t), \sigma(t)$ and $w(t)$, that are removed using the DWT scheme, as described in Section 4.

Finally, the method presented in this paper is a post-processing of the received data to validate the feasibility of the use of DWT in FM/FSO applications. The next phase of this work should be an FPGA implementation of the algorithm for a real time execution of the whole system in the receiver end.

\section{Author details}

Nader Namazi ${ }^{1}$, Ray Burris ${ }^{2}$, G. Charmaine Gilbreath², Michele Suite ${ }^{2}$ and Kenneth Grant ${ }^{3}$

1 Department of Electrical Engineering and Computer Science, Catholic University of America, Washington, USA

2 Naval Research Laboratory, Washington, USA

3 Defence Science \& Technology Organisation, Edinburgh, Australia

\section{References}

[1] Wilson B., Ghassemlooy Z., Darwazeh I., Analogue Optical Fiber Communications, IEE (1995).

[2] Chang W. S. C., RF Photonic Technology in Optical Fiber Links, (CUP, Cambridge, 2002). 
[3] Cox C. H. III, Analog Optical Links: Theory and Practice, (CUP, Cambridge, 2002).

[4] Refai H. H., Sluss J. J. Jnr, Refai H. H., Attiquzzaman M., "Comparative Study of the Performance of Analog Fiber Optic Links versus Free-Space Optical Links," Opt. Eng., (45)2, 025003-1 (2006).

[5] Bucholtz F., Burris H. R., Moore C. I., McDermitt C. S., Mahon R., Suite M. R., Michalowicz J. V., Gilbreath G. C., Rabinovich W. S., "Statistical Properties of a Short, Ana$\log$ RF Free-Space Optical Link," Proc. SPIE, Vol. 7324, Atmospheric Propagation VI, Edited by L. M. Wasiczko, G. C. Gilbreath, (SPIE, Bellingham, WA, 2009), p. 73240D-1.

[6] Refai H. H., Sluss J. J, Refai H. H., "The Use of Free-Space Optical Links for CATV Applications," Proc. SPIE, Vol. 5825, Opto-Ireland 2005, Edited by J. G. McInerney, G. Farrell, D. M. Denieffe, L. P. Barry, H. S. Gamble, P. J. Hughes, A. Moore, (SPIE, Bellingham, WA, 2005), pp. 408-15.

[7] Murphy J. L., Gilbreath G. C., Rabinovich W. S., Sepantaie M. M., Goetz P. G., “FMMRR Analog Audio System," Proc. SPIE, Vol. 5892, Free-Space Laser Communications V, Edited by D. G. Voelz, J. C. Ricklin, (SPIE, Bellingham, WA, 2005), p. 58921X-1.

[8] Rabinovich W. S., Mahon R., Burris H. H., Gilbreath G. C., Goetz P. G., Moore C. I., Stell M. F., Vilcheck M. J., Witkowsky J. L., Swingen L., Suite M. R., Oh E., Koplow J., “Free-Space Optical Communications Link at 1550nm Using Multiple-Quantum-Well Modulating Retroreflectors in a Marine Environment," Opt. Eng. (44)5, 056001-1 (2005).

[9] Grant K. J., Murphy J., Mahon R., Burris H. H., Rabinovich W. S., Moore C. I., Wasiczko L. M., Goetz P. G., Suite M. R., Ferraro M. S., Gilbreath G. C., Clare B. A., Mudge K. A., Chaffey J., "Free-Space Optical Transmission of AM Composite Video Signals using InGaAs Modulating Retro-Reflectors," Conference on Optoelectronic and Microelectronic Materials and Devices (COMMAD), University of Western Australia, December 2006.

[10] Grant K. J., Corbett K. A., Clare A. B., Davies J. E. \& Nener B. D., “Mitigation of Scintillation Noise by Common Mode Rejection," Proc. SPIE, Vol. 5793, Atmospheric Propagation II, Edited by C. Y. Young, G. C. Gilbreath, (SPIE, Bellingham, WA, 2005), pp. 106-117.

[11] Grant K. J., Clare B. A., Mudge K. A., Sprey B. M., Oermann R. J., "Real-Time Scintillation Noise Mitigation for Free Space Optical Transmission of Analogue and Digital Signals," Proc. SPIE, Vol. 6951 Atmospheric Propagation V, Edited by L. M. Wasiczko, G. C. Gilbreath, (SPIE, Bellingham, WA, 2008), p. 69510H-1.

[12] Grant K. J., Clare B. A., Martinsen W., Mudge K. A., Burris H. R., Moore C. I., Overfield J., Gilbreath G. C. \& Rabinovich W. S., "Free Space Optical Transmission of FM Audio/Video Signals using InGaAs Modulating Retro-Reflectors," Conference on 
Optoelectronic and Microelectronic Materials and Devices (COMMAD), Aust. National University, Canberra 2010.

[13] Grant K. J., Clare B. A., Martinsen W., Mudge K. A., Burris H. R., Moore C. I., Overfield J., Gilbreath G. C., Rabinovich W. S. \& Duperre J., “Laser Communication of FM audio/video Signals using InGaAs Modulating Retro-Reflectors," Proc. SPIE, Vol. 8038, Atmospheric Propagation VIII, edited by L. M. Wasiczko Thomas, E.J. Spillar, (SPIE, Bellingham, WA, 2011), p. 80380K-1.

[14] Grant, K.J., Mudge, K.A., Clare, B.A. \& Martinsen, W.M., "Ship-to-shore Free Space Optical Communications", Australian Institute of Physics Congress, Sydney, (2012).

[15] Burris H. R., Bucholtz F., Moore C. I., Grant K. J., Suite M. R., McDermitt C. S., Clare B. A., Mahon R., Martinsen W., Ferraro M., Sawday R., Xu B., Font C., Thomas L. M., Mudge K. A., Rabinovich W. S., Gilbreath G. C., Scharpf W., Saint-Georges E., Uecke S., "Long Range, Analog RF Free Space Optical Communication Link in a Maritime Environment," Proc. SPIE, Vol. 7324 Atmospheric Propagation VI, Edited by L. M. Wasiczko, G. C. Gilbreath, (SPIE, Bellingham, WA, 2009), p. 73240G-1.

[16] Ziemer R. E. and Tranter W. H., Principles of Communications, Systems, Modulation and Noise, (5th Edition, John Wiley \& Sons, Inc., 2002).

[17] Rao R. M. and Bopardikar A. S., Wavelet Transforms: Introduction to Theory and Applications, (Addison Wesley Longman, Inc., 1998). 